\title{
ESTUDIO PRELIMINAR SOBRE LA BIOLOGÍA DE LAS POBLACIONES DE GELIDIUM CANARIENSIS (GRUNOW) Seoane en Gran Canaria
}

\author{
María José BETANCORT VILLALBA \& M. Nieves GONZÁLEZ HENRIQUEZ
}

RESUMEN: Se han realizado los trabajos preliminares sobre la biología de Gelidium canariensis (Grunow) Seoane en tres localidades de la costa Norte de Gran Canaria, tipificando las distintas clases de talla y comparando las distintas poblaciones utilizando los parámetros biomasa y número de individuos por clase de talla.

Palabras clave: Gelidium canariensis, Biología, Canarias.

SUMMARY: A preliminary study on the biology of Gelidium canariensis (Grunow) Seoane was made in three places on the North coast of Gran Canaria Island. We used four size classes making a between-population comparison using parameters biomass and number of members per size king.

Key words: Gelidium canariensis, Biology, Canarias.

\section{INTRODUCCIÓN}

La importancia de este estudio radica en que Gelidium canariensis (Grunow) Seoane es una especie agarofita cuya distribución está restringida a las costas de Canarias (Lám. 1a). Es un alga que se localiza en latitudes subtropicales por lo que el comportamiento de las poblaciones naturales probablemente será diferente al de otras especies del género de latitudes más altas ya estudiadas en la Península Ibérica (Gelidium sesquipedale (Clemente) Thuret) (Salinas \& al., 1976; Requena \& al., 1978; Oliveira, 1984), de esta forma tendríamos un punto de comparación entre unas y otras.

El principal objetivo de este trabajo es analizar la biomasa, densidad y variación de los parámetros biométricos dentro de cada población. Con este muestreo previo se conocerá la variabilidad intrínseca para un determinado parámetro en dichas poblaciones. 


\section{MATERIAL Y MÉTODOS}

A lo largo de la costa de Gran Canaria se han seleccionado tres estaciones (Caleta Arriba (N), Boca Barranco (N) y Puerto de Las Nieves (Agaete, NO) donde la distribución de las poblaciones de Gelidium canariensis (Grunow) Seoane es más homogénea para realizar este tipo de estudio.

Parte de la metodología va a depender en gran medida de la localización de las poblaciones a estudiar (Salinas \& al., 1976; Gorostiaga \& al., 1987; Cos Asensio \& al., 1987). Al ser poblaciones de límites de marea lo más cómodo es trabajar con muestreos destructivos. Para este estudio preliminar se han realizado las medidas de los parámetros en una franja de $100 \times 50 \mathrm{~cm}$. Esta superficie ha sido recogida mediante un cuadrado de PVC de 50 x $50 \mathrm{~cm}$ subdividido en cuatro unidades de $25 \times 25 \mathrm{~cm}$ (se recogieron dos cuadrados consecutivos en la misma franja de costa) en Abril de 1988.

El material recogido se separó en el laboratorio para agrupar las dintintas clases de talla y comparar las tres estaciones.

Se procedió a pesar y secar el material para obtener la biomasa de cada clase de talla y estación. El material se secó en una estufa a $80-100^{\circ} \mathrm{C}$ durante tres días hasta obtener el peso constante.

\section{RESULTADOS}

El material de las superficies muestreadas se pesó en el laboratorio (peso fresco bruto) y posteriormente se separaron los individuos de las matas para clasificarlos, llegándose a la conclusión de que los individuos de las poblaciones se pueden clasificar en cuatro clases de talla: de $0-5 \mathrm{~cm}$ talla inferior, de $5-10 \mathrm{~cm}$ talla media, de $10-15 \mathrm{~cm}$ de talla grande y de $>15 \mathrm{~cm}$ talla superior (Lám. 1b). Una vez separados los individuos se pesaron por clase de talla (peso fresco). La diferencia obtenida corresponde a algas epífitas, pequeñas piedras, granos de arena, conchas, tubos de poliquetos, sipunculidos, cangrejos etc (tabla 1). (Lám. 1c y d).

\begin{tabular}{lccc}
\hline & (g) PESO FRESCO BRUTO & PESO FRESCO & DIFERENCIA \\
\hline AGAETE & 2470.59 & 1869.20 & 601.37 \\
B. BARRANCO & 2356.44 & 1718.45 & 564.70 \\
CALETA & 2800.85 & 1912.39 & 888.46 \\
\hline
\end{tabular}

Tabla 1: Datos de peso fresco bruto y peso fresco. 

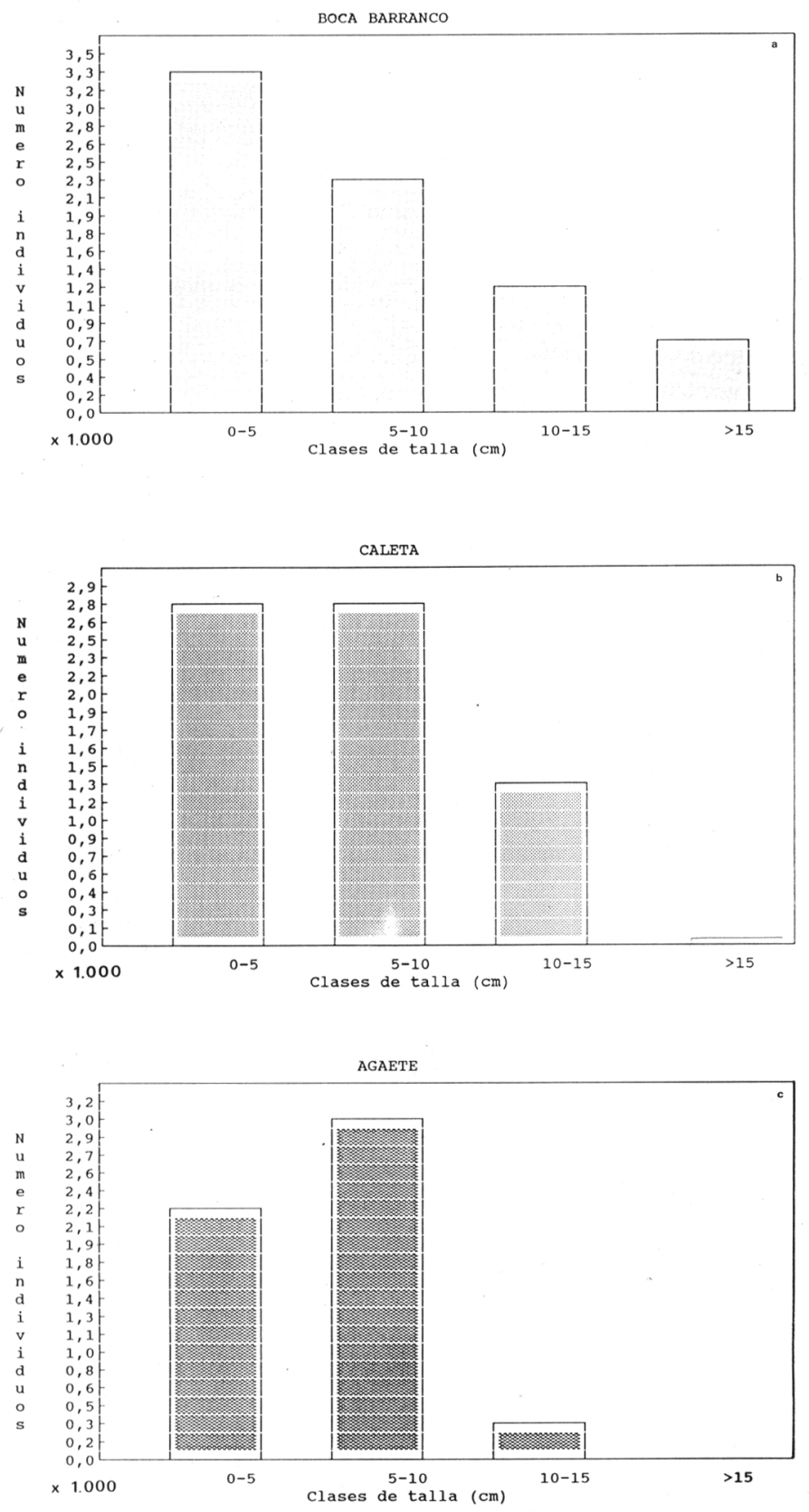

Fig. 1. Número de individuos por clases de talla; a) en la localidad de Boca Barranco; b) en la localidad de Caleta Arriba; c) en la localidad de Agaete. 
A la vista de los resultados obtenidos, en la que se analiza el número de individuos por clases de talla y por población, se puede observar que la población más homogenea y en la cual están representadas todas las clases de talla (fig. 1a) es la de la estación de Boca Barranco; la estación de Caleta situada también en el Norte de la isla, aun teniendo representantes de todas las clases de talla, la población es menos homogénea siendo muy abundantes los individuos de tallas inferior y media $(0-5,5$ $10 \mathrm{~cm}$ ) (fig. 1b); la otra estación situada el NO de la isla (Puerto de Las Nieves) no tiene individuos de talla superior $(>15 \mathrm{~cm}$ ) (fig. 1c) siendo una población homogénea pero de talla media $(5-10 \mathrm{~cm})($ tabla 2$)$.

\begin{tabular}{lccccc}
\hline & $0-5$ & $5-10$ & $10-15$ & $>15$ & Total \\
\hline AGAETE & 2163 & 3116 & 293 & - & 5572 \\
CALETA ARRIBA & 2803 & 2846 & 1313 & 25 & 6987 \\
BOCA BARRANCO & 3466 & 2233 & 1235 & 760 & 7694 \\
\hline
\end{tabular}

Tabla 2: Número de individuos por clases de talla y por localidades.

Si comparamos las tres estaciones, las situadas (fig. 2) en el $\mathrm{N}$ tienen mayor número de individuos por clases de talla qué la del NO.

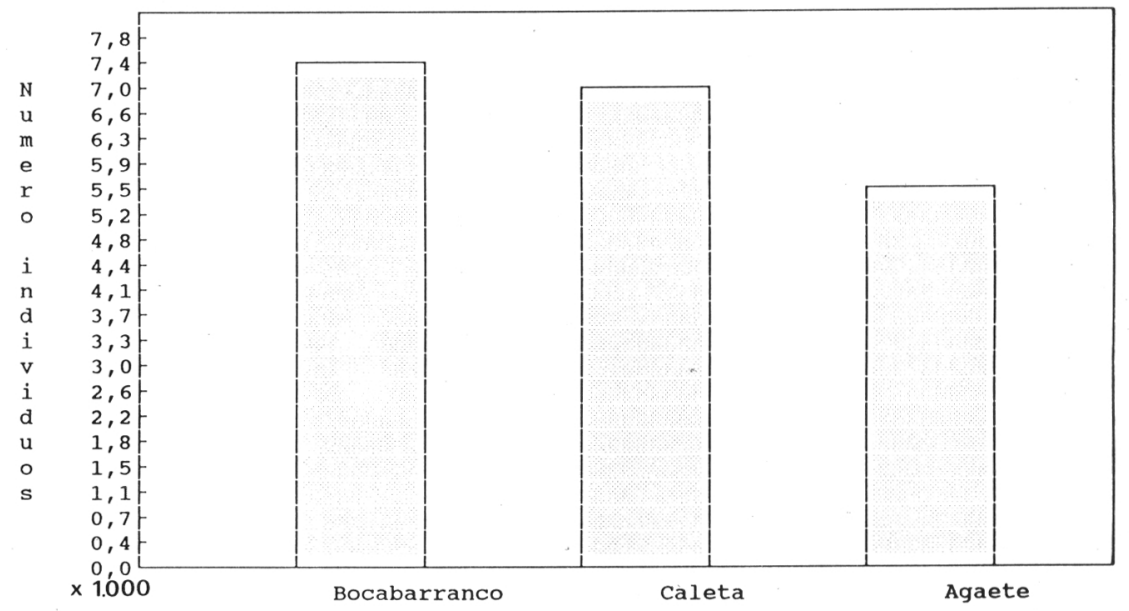

Fig. 2. Número de individuos totales por localidades.

Comparando los datos de números de individuos por clases de talla conjuntamente en las tres estaciones (fig. 3) vemos que el valor máximo se va rodando 
dependiendo de la estación; así observamos que Boca Barranco va disminuyendo paulatinamente su valor a medida que incrementa su tamaño, en Caleta estabiliza su máximo en las dos primeras clases de talla, mientras que en Agaete es verdaderamente importante la clase media. Con esto se deduce que la regeneración, manifestada en el alto índice de individuos de la talla inferior, es mayor en la estaciones del $\mathrm{N}$ que en la del NO, mientras que la clase media es superior en la estación del NO, quizás sea debido al efecto del oleaje que incide mucho más en esta clase de talla en las estaciones que están al norte de la isla.

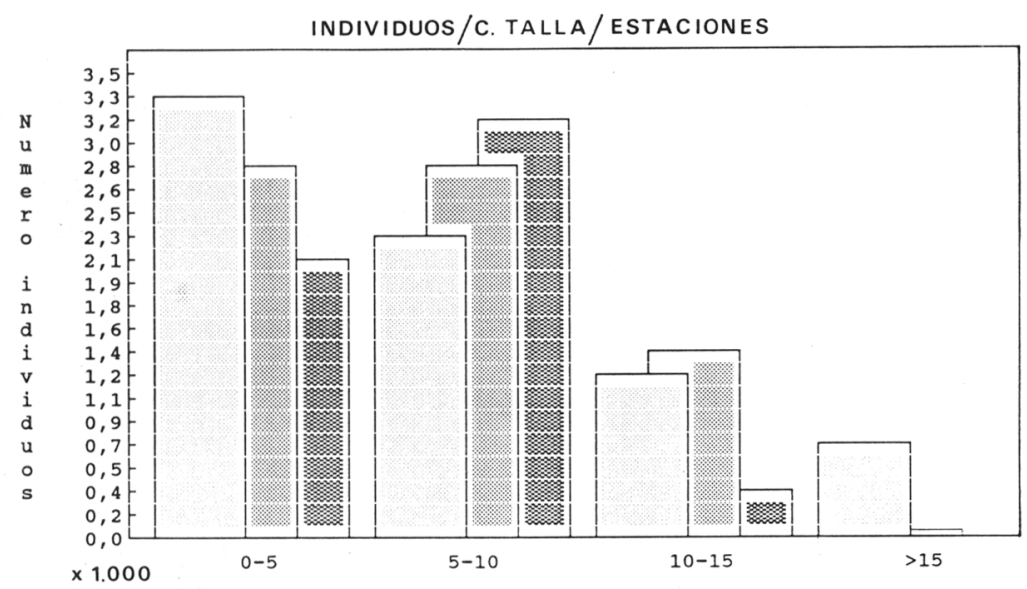

Fig. 3. Comparación de números de individuos por clases de talla y por localidades; el entramado corresponde a la simbología representada en la fig. 1.

Si analizamos los datos de biomasa, en Boca Barranco (fig. 4a) la talla de 0-5 $\mathrm{cm}$ es la de menor valor y la mayor es la de talla $>15 \mathrm{~cm}$, es decir, a mayor tamaño mayor valor de la biomasa, en Caleta (fig. 4b) la de mayor valor de biomasa es la de 10-15 cm y sólo en Agaete (fig. 4c) se mantiene como mayor la de 5-10 cm (tabla 3).

\begin{tabular}{lcccrc}
\hline & $0-5$ & $5-10$ & $10-15$ & $>15$ & \multicolumn{1}{c}{ Total } \\
\hline AGAETE & 24.37 & 359.58 & 74.98 & - & 458.93 \\
CALETA ARRIBA & 27.76 & 259.70 & 301.91 & 13.81 & 603.18 \\
BOCA BARRANCO & 29.11 & 107.63 & 147.68 & 175.95 & 460.37 \\
\hline
\end{tabular}

Tabla 3: Biomasa (peso seco en g) por clases de talla en las localidades estudiadas.

Si comparamos la biomasa total de cada estación (fig. 5) la mayor es Caleta aunque, sin embargo, la diferencia con las otras dos estaciones no es excesivamente significativa. 

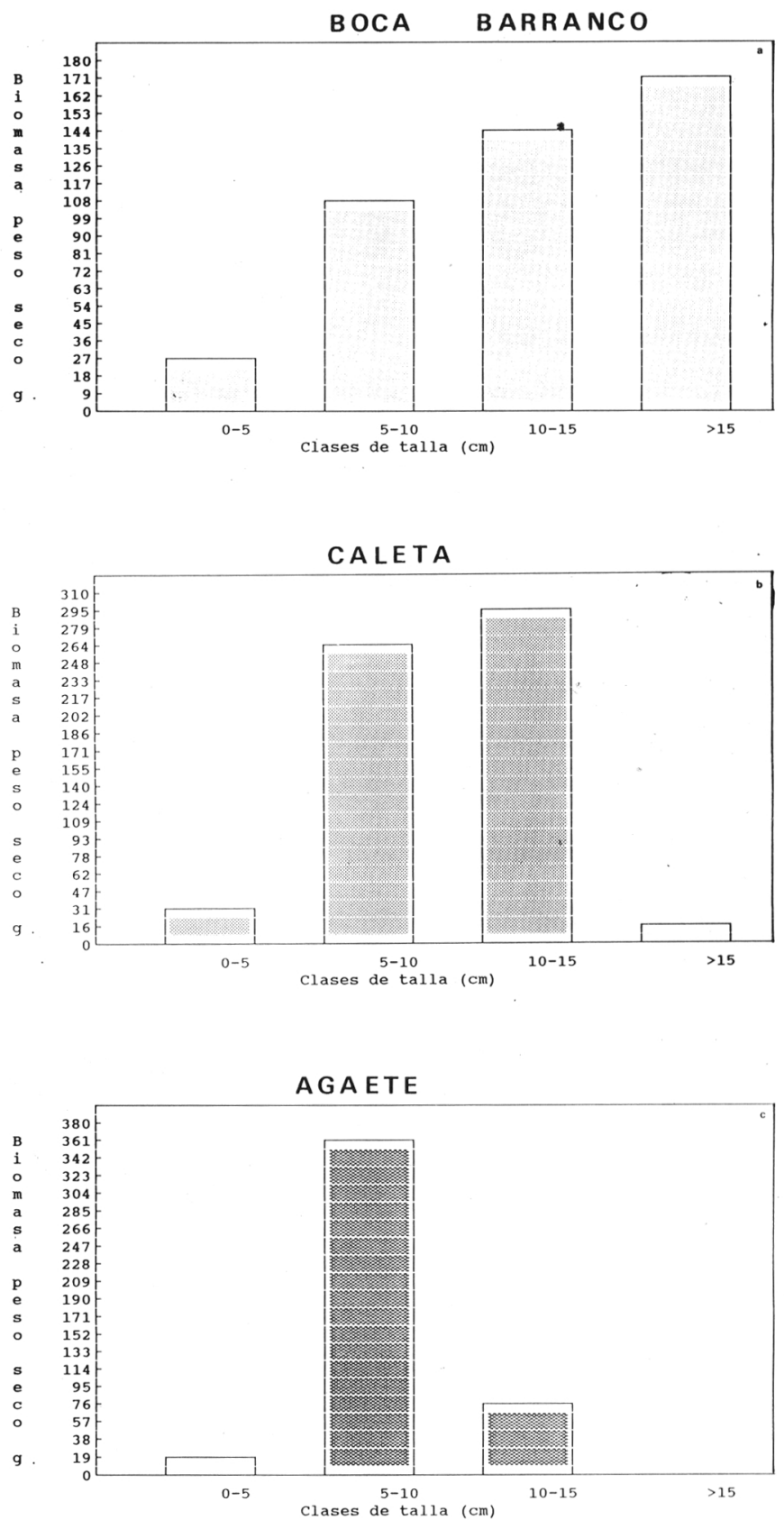

Fig. 4. Biomasa por clases de talla; a) en la localidad de Boca Barranco; b) en la localidad de Caleta Arriba; c) en la localidad de Agaete. 
Analizando los datos de biomasa por clases de talla y localidades (fig. 6) se observa que son inversas a la de números de individuos por clases de talla y localidades en Boca Barranco, ya que la biomasa de la talla pequeña es muy poca siendo más importante la de tallas superiores. En Agaete se mantiene el valor máximo en la talla media mientras que las demás son poco relevantes. En Caleta, las tallas media y grande se reparten los valores más altos aportando poca biomasa las restantes.

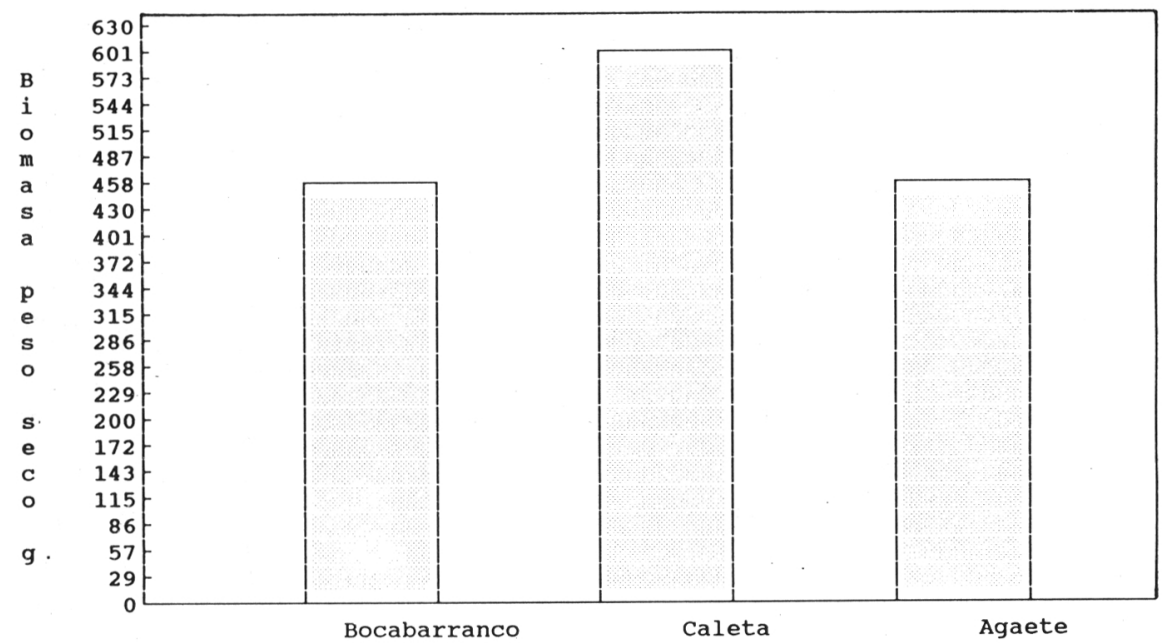

Fig. 5. Biomasa total por localidades.

BIOMASA/C. TALLA/ESTACIONES

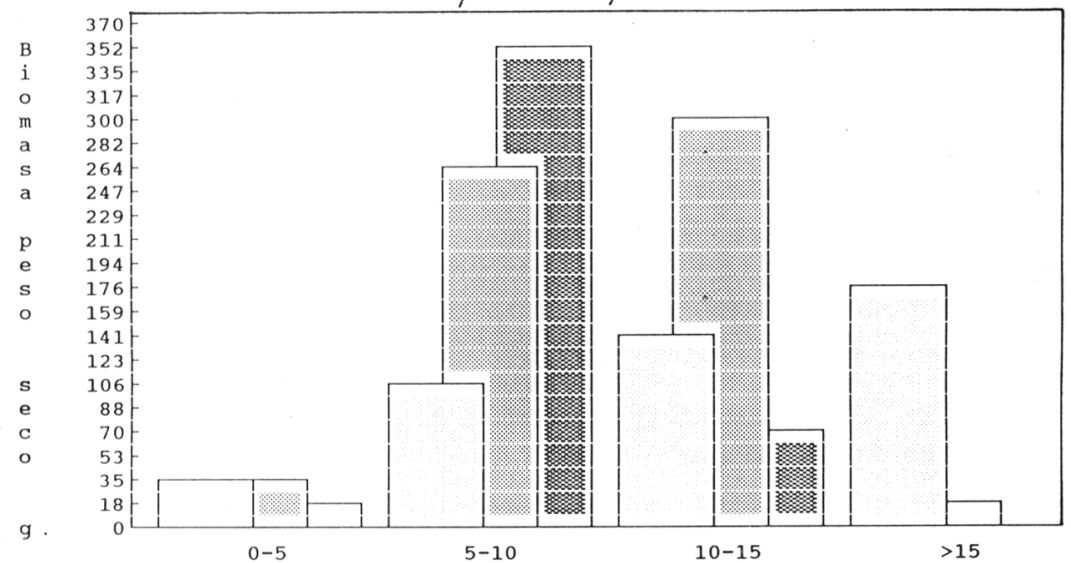

Fig. 6. Comparación de la biomasa por clases de talla en las localidades estudiadas; la simbología del entramado corresponde a la representada en la fig. 1. 

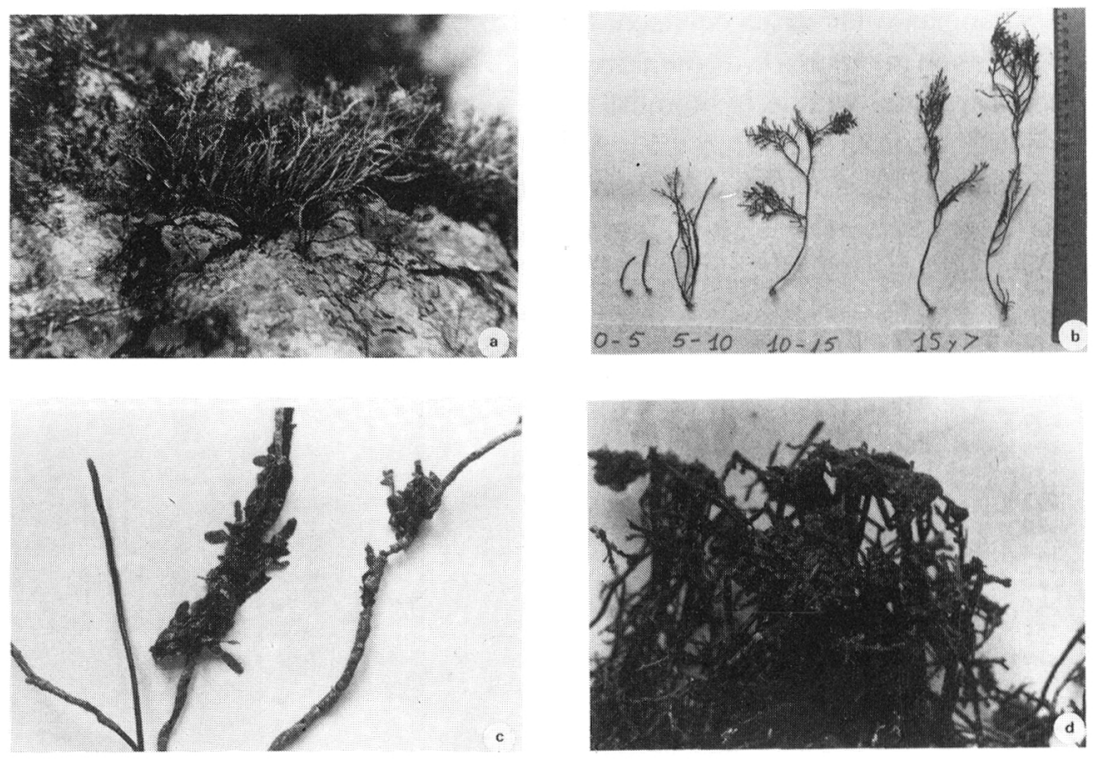

Lámina 1. a) Matas de Gelidium canariensis (Grunow) Seoane en el medio natural; b) ejemplo de las diferentes clases de talla que se han encontrado; c y d) epífitos del talo y base en Gelidium canariensis (Grunow) Seoane.

\section{BIBLIOGRAFÍA}

COS ASENSIO, C. \& M.A. SIGUAN -1989- Estudio biológico de Pterocladia capillacea (Gmelin) Born et Thur. Crecimiento de la planta in situ. Anales del Jardín Botánico de Madrid, 46-1:47-54.

GOROSTIAGA, J.M., K. AURREKOETXEA \& A. PASCUAL -1987- Tasa de elongación apical en Gelidium sesquipedale. Resúmenes del VII Simposio Nacional de Botánica Criptogámica.

OLIVEIRA, J.C. -1984- Notas sobre o creccimento de Gelidium sesquipedale (Clem.) Born et Thur., na costa ocidental portuguesa. Actas do IV Simposio Iberico de Estudos do Benthos Marinho. Lisboa. Volume III: 51-62.

REGUERA, B., J.M. SALINAS \& R. GANCEDO -1978- Biometría en Gelidium sesquipedale (Rodophyta). Boletín del Instituto Español de oceanografía. II parte. № 255.

SALINAS, J.M., B. REGUERA RAMIREZ \& R. GANCEDO - 1976- Biometría en Gelidium sesquipedale (Rodophyta). Boletín del Instituto español de Oceanografía. I parte. № 226.

(Aceptado para su publicación en Junio de 1.990 )

Dirección de los autores: Jardín Botánico Canario «Viera y Clavijo». Apartado 14 de Tafira Alta. 35017 Las Palmas de Gran Canaria. 